\title{
Euclid's Error: Non-Euclidean Geometries Present in Nature and Art, Absent in Non-Higher and Higher Education
}

\author{
Cristina Alexandra Sousa \\ Universidade Portucalense Infante D. Henrique, Portugal
}

\begin{abstract}
This analysis begins with an historical view of Geometry. One presents the evolution of Geometry (commonly known as Euclidean Geometry) since its beginning until Euclid's Postulates. Next, new geometric worlds beyond the Fifth Postulate are presented, discovered by the forerunners of the NonEuclidean Geometries, as a result of the flaw that many mathematicians encountered when they attempted to prove Euclid's Fifth Postulate (the Parallel Postulate). Unlike what happens with the initial four Postulates of Euclid, the Fifth Postulate revealed a lack of intuitive appeal, and several were the mathematicians who, throughout history, tried to show it.

After this brief perspective, a reflection is made on the presence of Non-Euclidean Geometries in Nature and Art and, yet on this subject, some philosophical implications.

Then, it is analyzed the study of Geometry in Portuguese Secondary Education and the absence of the study of Non-Euclidean Geometries in Higher Education curricula in Portugal. Finally, some suggestions will be made on the inclusion of NonEuclidean Geometries in some details of the curricula of Geometry in Non Higher Education.
\end{abstract}

\section{Introduction}

Since the dawn of time, Man has always been concerned to understand the world he lived in. He observed carefully and accurately the shapes of nature and felt the need to theorize, and later, to find mathematical proofs for various empirical elements.

If we examine with some attention, in fact, we have a daily need to use Geometry. When we say something is far away, we are referring to a length and this is Geometry. When we discuss about the area of a football field, we use Geometry. If we say that a dress is wide, we are thinking of volumes, and therefore thinking in Geometry. Geometry accompanies us all the time.

One of the most important books ever written is probably Euclid's Elements. Its volumes have provided a model for the rigorous development of mathematical ideas, which is still used today. The Euclidean Geometry defines the situations of the plan. However, when we are dealing with different surfaces, we are faced with the impossibility of solving problems through the same geometry.

Unlike what happens with the initial four postulates of Euclid, the Fifth Postulate, the famous Parallel Postulate, revealed a lack intuitive appeal, and several were the mathematicians who, throughout history, tried to show it. Many retreated before the findings that this would be untrue, some had the courage and determination to make such a falsehood, thus opening new doors to Geometry.

One puts up, then, two questions. Where can be found the clear concepts of such Geometries? And how important is the knowledge and study of Geometries, beyond the Euclidean, to a better understanding of the world around us? The study, now developed, seeks to answer these questions.

Since the primary objective is a response to these earlier questions, this study is divided into three phases. The first phase focuses on the historical evolution of Geometry, from its beginnings to the work of the Greek Euclid. In a second phase, the main precursors of Geometry are presented and, subsequently, the discoverers of Non-Euclidean Geometries, the Elliptic and Hyperbolic Geometries themselves, being the most outstanding among all the Non-Euclidean, and even some models of its representations. The third and final phase is related to the analysis of the presence of Non-Euclidean Geometries in Art and in the Real, the study of Geometry in Secondary Education and NonEuclidean Geometries in Higher Education, ending up with some philosophical implications that one understands be relevant, given all the controversy generated around these Non-Euclidean theories of Geometry.

\section{Geometry: From its Origins to Euclid}

It is impossible to follow the early evolution of Geometry but it is assumed that, from the observation of Nature, Man created the concept of forms, figures, bodies, volumes and distances.

In the absence of documentary evidence, it is impossible to track the early evolution of Geometry, but it is nonetheless interesting to speculate what perhaps was past, for the simple fact of finding prehistoric paintings or even simple "risks", which shows that Man, since he came to think as Man, was always concerned with the representations he 
observed, was a religious sense, was an aesthetic sense of fun, was one way to mark their land, was in an explanatory sense, which might be the one closer to scientific discovery as we consider it today.

\subsection{Mathematics in Greece}

In contrast to the Babylonians and Egyptians, the Greeks used the logical demonstration at the expense of experimentation by trial and error, deduction instead of induction. The Greeks also developed the first comprehensive scientific theories about the heavens, i.e., Astronomy began, of course, with the aid of Geometry and reasoning often in geometric bases.

Some geometric results are also attributed to the Pythagoreans. The most famous is the theorem of Pythagoras himself, a key theorem of Euclidean Geometry. It is also supposed to have been the Pythagoreans who discovered what today we learn about triangles, parallel lines, polygons, circles, spheres and regular polyhedra. They knew, in particular, that the sum of the angles of a triangle was $180^{\circ}$. A theory of limited and similar figures that the plan can be filled with equilateral triangles, squares and regular hexagons are included among their results.

So, it was in Greece that geometrical concepts have acquired a scientific form, achieving its splendour with Euclid.

Euclid's Elements, one of the most important works of all time, contains the teachings which are still the basis of modern Geometry. Euclid's Elements, with an exceptional importance in the history of Mathematics, does not present Geometry as a mere cluster of unconnected data, but rather as a logical system of definitions, axioms and postulates. The Elements is based on five logical postulates. Four of these postulates seem to come accordingly to our experience. However, the Fifth Postulate does not sound so obvious and intuitive, and is considered a Euclid's own invention. One might remember the Postulate by the Axiom enunciated by Hilbert: for a point outside a given line there is one and one only straight line parallel to it.

This is the point of controversy that led to centuries of discussion in the scientific community and served as the motto for this particular work.

\section{New Geometric Worlds beyond the 5th Postulate}

\subsection{The Forerunners of Non-Euclidean Geometries}

Several mathematicians tried to prove the correctness of Euclid's 5th Postulate for a long time. Although they could get close to real conclusions, they failed, as its primary objective was to prove the Postulate, and not conclude that this could be false (Saccheri, Legendre, Farkas Bolyai, Gauss). Moreover, even with assurances regarding their results, the fear of facing the mathematical community, and the shame of being marginalized by their act of courage, always stopped them from publishing such findings.

As Greenberg said, it is delightfully instructive to observe the mistakes made by capable people as they struggled with the strange possibility that they or their culture might not accept their conclusions.

It was finally with the courage and determination of János Bolyai and Nikolai Lobachevsky that the Non-Euclidean Geometries were finally acknowledged in Mathematics. Although working separately, both mathematicians developed a new Geometry that exists in spaces of constant negative curvature, which became known as Hyperbolic Geometry.

Later, Bernhard Riemman also developed a similar Geometry to the one of Bolyai and Lobachevsky, creating the concept of Elliptic (or Spherical) Geometry, which exists in areas of constant positive curvature. With his studies, Riemann influenced profoundly Geometry and Analysis and presented, a half-century before, the solutions of geometrical spaces designed by Albert Einstein to the Theory of Relativity, which only with such results became possible, thus contributing to the development Physics.

So, let's see how we can define each one of these Geometries.

\subsection{Hyperbolic Geometry}

The Hyperbolic Geometry exists in spaces of constant negative curvature. Let's think of all the hyperbolic space as being contained in a disk. To condense an infinite world inside a disk, inevitably, leads to expected distortions. We shall consider the centre of the disk in a similar manner to that of Euclidean Geometry. However, the closer we are to the limit of the disk, the greater is the distortion obtained in the represented images. We then have to think of the edge of the disk as being infinitely distant from the centre of the disc. This means that

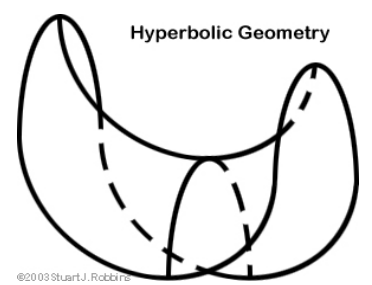

Figure 1. Hyperbolic surface everything that we see near the boundary of hyperbolic space will appear vastly smaller than it actually is.

The fundamental difference between this and the Euclidean Geometry is that the Parallel Postulate is 
replaced by the Hyperbolic Axiom that says that for a point outside a given line there is a multitude of straight lines parallel to it.

It is also known that in the hyperbolic surface the sum of the internal angles of a triangle is always less than $180^{\circ}$ and there are no squares or rectangles, since the sum of its interior angles is less than $360^{\circ}$.

\subsection{Elliptic Geometry}

On the other hand, if the surface has constant positive curvature, i.e., is spherical, and since the lines are geodesics on the surface (such as the globe's longitude lines), they always meet in two points (the poles), with no place for parallel lines. Again falls to the ground the validity of the Parallel Postulate.

In these surfaces, the sum of the internal angles of a triangle is always greater than $180^{\circ}$.

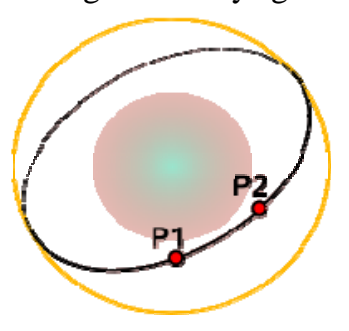

Figure 2. Elliptic Surface
Although not theorized, the Spherical, or Elliptic, Geometry was already for a long time known as the Geometry of the Offshore Sailor, since he moved from one point to another along the line of shortest distance between two points, or wandering through a range of geodesic.

The work of Riemann allowed that numerous Geometries could be design, each associated to a given surface and a corresponding metric, determined by the curvature of the surface in question.

\section{The Presence of Non-Euclidean Geometries in the Real and its Absence in the Study of Geometry}

If we look at a basketball ball, for example, we concluded that it is a sphere. However, for an ant or a fly that moves on its surface, it always seems flat. Indeed, both insects on the ball, as humans on Earth's surface, are not able to check the curvature of the surface where they are observed when the scales are so disparate. If there are no precise measurements and we do not have full knowledge of the areas that we look down, we cannot draw any conclusions. about the whole.

Thus, we are now arrived to the last part of this work where will be analysed the presence of the Non-Euclidean Geometries in the Real and its absence in the study of Geometry.

\subsection{The Application of Non-Euclidean Geometries in Artistic Expressions}

What can we mean by Art? This is an issue that depends on the place and time where we stand. All people on Earth share a common aspiration to create, in order to achieve the ultimate truth and the use of technology. To a certain extent, any human activity can be considered an Art form and, then, we must decide what considered "artistic" and what not.

We can, now, raise the misleading question of whether or not is Art a uniquely human activity.

We are faced by many phenomena in Nature considered as authentic forms of Art. A crucial

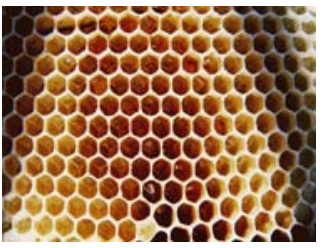

Figure 3. Honeycomb difference between human Art and animal Art is the intention with which it is created. The animals create what seems to be more practical and functional to them, as is the case of bees and their honeycombs, for example (Figure 3). Humans create it just for aesthetic enjoyment.

The opposition between Art and Science can be seen as artificial and, in a more positive note, we consider Art as a driving force of Science.

There are names in Art History that are synonymous of the combination of Art and Science. As an example, one can refer the great Renaissance artist, Leonardo Da Vinci. Da Vinci produced thousands of drawings where artistic and scientific investigations meet. As Leonardo was a convinced Christian, he believed that Art and Science were two paths that lead to a single destination, the ultimate spiritual truth.

A compelling and emblematic figure in the form of artistic expression, as a combination of mathematical, geometric and physically impossible concepts was M. C. Escher. He became par excellence the creator of impossible worlds.

In particular, the regular division of a surface facilitated his approach to infinity. This surface is no more than one model of representation of the hyperbolic disk (Figure 4).

Escher, during his work, developed a true glorification of

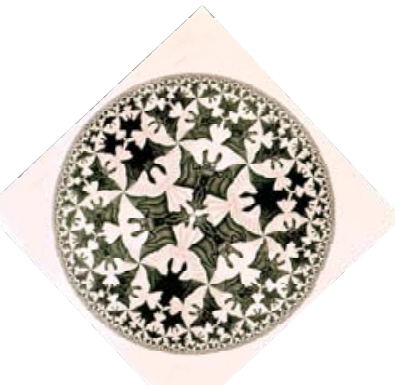

Figure 4. Circle limit IV by M. C. Escher reality, interpreting mathematically patterns and rhythms of Nature forms.

But of all forms of Art, the one which is more closely related to Geometry is undoubtedly Architecture. The architectural structures are the 
result of geometric principles and the final result may come to be a solid figure. Even before the advent of Euclidean Geometry, mankind built houses, monuments and cities even now resorting to geometric figures that have subsequently led to Euclidean Geometry itself.

Despite the great speed of advancement of societies and technology and the demand for new architectural styles, Euclidean Geometry is still present in most buildings. However, newer concepts from the Non-Euclidean Geometries began to contribute to changes. To this end, new programs and computational tools have been a valuable aid for the trial and execution of new and bold architectural forms.

Antoni Gaudí can, in this field, be considered the great master of Organic Architecture that is the Architecture which is based on principles and forms based on Nature. The organic volumes used by him were the result of structural parts which we refer to natural forms, both animal and vegetable. Therefore, we can find the Non-Euclidean Geometries in the architectural work of Gaudí, indirectly, because of his inspiration in natural forms. Gaudí will forever be an icon of world Architecture.

\subsection{The Presence of Non-Euclidean Geometries in the Real}

It is now time to look at geometrical structures independent of human intervention.

Taking into account the knowledge of common sense, easily we assume that the Geometry of Nature is Euclidean. However, there is no basis for this, partly because it was never possible to prove the Fifth Postulate of Euclid, since it wasn't very clear. The Fifth Postulate is a merely detail of Absolute Geometry and there is no reason or evidence to suggest that Euclidean Geometry should be the basis for the choice of Nature. Also concepts such as infinity have no place in the real world, so that, in itself, brings us to abstract concepts.

As referred before, there are in Nature an infinite number of elements that cannot be defined by traditional Euclidean Geometry. Examples of that are some trees, clouds, mountains, rivers, the system of blood vessels, nerve structures, etc., which have the name of fractals (Figure 5).

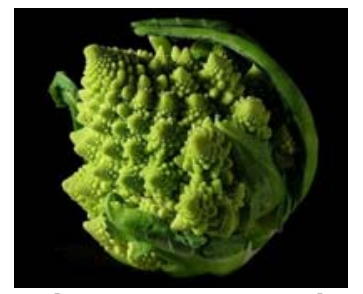

Figure 5. Broccoli
With greater or lesser degrees, these elements are classified into various magnitudes. But although they are present throughout Nature, they did not deserve further study until the early 20th Century.

Now, a figure of Mathematics and Physics that cannot be forgotten is Albert Einstein. Since the pioneer work of Einstein, we now have a different view of space and time. For Einstein the Universe was not flat nor time was absolute and both were combined in a curved space-time. The particularity of his work was to have added to the already known three dimensions (width, height and depth), the dimension of time. His theoretical work has allowed the development of atomic energy, although it does not foresee such a possibility.

Another issue raised by Einstein was to call attention to the fact that physical space has curvature. When we work in a flat space, the shortest distance between two points, for example, is determined by a line segment that joins them. In curved space things are processed differently, as indeed has already been discussed earlier.

Let's now consider a problem, perhaps already known to all.

"From a certain point on Earth, a hunter walked $10 \mathrm{~km}$ to the South, $10 \mathrm{~km}$ to the East and $10 \mathrm{~km}$ to the North, thus returning to the starting point. There he found a bear. What colour was the bear?"

At first glance it might seem impossible. However, since it is known by all that the Earth is not flat, the solution is imminent. If the three

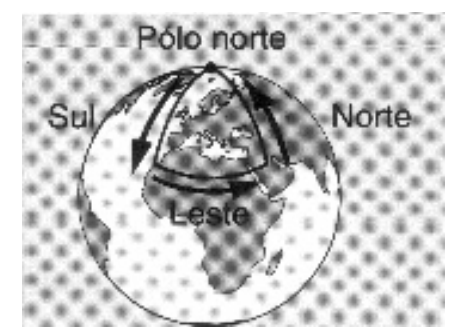

Figure 6. Hunter's Path movements of the hunter are perpendicular to each other and he returns to the starting point, he can only be found in one of Earth's poles, as we can easily see by figure 6 . If he encountered a bear, it can only be white and the location is the North Pole.

\subsection{Analysis of the Study of Geometry in Secondary School}

We can now say that there is no doubt about the presence of Non-Euclidean Geometries in the world. Also notable is its use by various artists. For this reasons, one tried to investigate the "state of play" on the study of Geometry in Secondary Education and of Non-Euclidean Geometries in Higher Education in Portugal.

The general perception in public opinion that students are required to devote more time to Mathematics because of broad disciplinary curricula and corresponding workload is a false one. Rather, the syllabus steadily decreases both the amount of subjects and their difficulty. For its part, the study of Geometry suffers the consequences, as it is repeatedly put on hold and, when studied, there is no depth or consistency and, therefore, no logical understanding by students. 
As a result of all these factors, when students are confronted with the necessity of working the materials in a more autonomous way, one verifies that they have not this ability yet mastered, which compromises the acquisition to more accurate and comprehensive knowledge.

Geometry, as an integral and forming discipline of Mathematics Portuguese curricula, seems somewhat helpless, abandoned and relegated to a lower level, needing to be rescued by the School and all teachers.

\subsection{The Non-Euclidean Geometries in the Study Plans of Higher Education}

In addition, in University Education students don't have, in most cases, any knowledge of Geometries other than Euclidean. The reverse situation could positively influence the way students comprehend the world around them, by broadening their geometrical horizons.

Concerning to the study of Non-Euclidean Geometries in Higher Education, there was done a demonstrative analysis, although not exhaustive, of the presence of Non-Euclidean Geometries in the curricula at senior level in Portuguese Universities. In about two dozen of courses, only five contained references to the study of Non-Euclidean Geometries.

It is a bit unacceptable the absence to its study even in courses of Mathematics, as occurs in the University of Coimbra and the University of Minho.

And it is also unacceptable that in all the courses analyzed, only seven had references to the study of traditional Geometry. This study is absent in courses that could benefit greatly by its presence, as is the case of Arts and Civil and Environmental Engineering.

This apparent negligence of the study of NonEuclidean Geometries may be negative not only in a scientific level but also ruling to the training of mental structures of perception of space and the world. As previously one tried to exemplify, the Non-Euclidean Geometries are present in the Real around us in Nature and Art.

\subsection{Philosophical Implications}

Certainly, Engineering and Architecture are evidence that Euclidean Geometry is extremely useful in measuring common distances when they are not too extensive. However, the accuracy in the representation of Euclidean Geometry is less when dealing with longer distances.

According to Einstein, space and time are inseparable and the Geometry of space-time is affected by matter, so light rays are in fact bent by the gravitational attraction of masses. Space is no longer conceived as an empty Newtonian box whose contours are not affected by stones placed within. The problem is now much more complex than Euclid or Lobachevsky ever imagined - none of their Geometries is adequate for our present concept of space. But this does not diminish the historical importance of Non-Euclidean Geometries.

Thus, Geometry is purely a formal exercise of the deduction of certain conclusions from the formal assumptions. Mathematicians make claims but that tells us nothing about the meaning of the truth of the hypotheses. The primitive notions that appear in the hypotheses are implicitly defined by the axioms, rules as they are, telling us how to play the game.

\section{Conclusion}

Euclid is, arguably, the most famous geometer of all time, having been through his window that Geometry has always been contemplated. To him we owe the birth of abstraction, the concept of proof and deductive reasoning itself.

One aims a Mathematics Education that enables students to analysis, discussion, conjecture, appropriation of concepts and formulation of ideas and for that to happen, students must be motivated and interested in Mathematics classes. It is believed that Non-Euclidean Geometries can assist with this motivation and interest, since they have something that is part of the daily student. The obvious negligence in the study of Non-Euclidean Geometries is, in our opinion, negative not only in Science but also harmful to the level of training of the mental perception of space and world. The NonEuclidean Geometries are present in the Real around us, in Nature and Art, as previously one tried to exemplify.

In a simple and clear way, one tried to demonstrate that, despite the popularized idea that Euclidean Geometry is the only current Geometry in the world, the Non-Euclidean Geometries are present in numerous events that surrounds us. It is expected, therefore, the generalization of knowledge on these issues so that we can look at the reality in a more complete way.

But despite the logical shortcomings of Euclid's work in light of current standards, and in spite of the critical movements started at the end of the 17th Century, which culminated in the proposed alternative Geometries and a complete review of the fundamentals of Euclidean Geometry and the emergence of new concepts about the classification of Geometries, none of that takes the value of his monumental work.

In the same way as the Euclidean concepts were questioned over the centuries, but not their validity, will the new Non-Euclidean Geometries one day come to be superseded by new theories, the fruit of tireless research and human curiosity? 
According to the philosopher Immanuel Kant, the reality is synthesized in accordance, not with the real world, but with our mind and its limitations.

But as Russell said, Mathematics is the discipline that we don't know what we are talking about nor whether what we say is true...

\section{References}

[1] BONOLA, Roberto, CARSLAW, H. S., Non-Euclidean Geometry: A Critical And Historical Study Of Its Development. Authorized English translation with additional appendices by H. S. Carslaw. New York, Dover Editions, 1955. (ed. orig.: 1912) (reimpr. Kessinger Publishing 2008).

[2] DAVIS, Philip J., HERSH, Reuben, Descartes' Dream: The World According to Mathematics. London, Penguin Books, 1990 (ed. orig.: Harcourt Brace Jovanovich, c1986).

[3] DAVIS, Philip J., HERSH, Reuben, A Experiência Matemática. Lisboa, Gradiva, 1995 (ed. orig.: Birkhäuser Boston 1981).

[4] ERNST, Bruno, O Espelho Mágico de M. C. Escher. TASCHEN GmbH, 2007 (ed. orig.: 1978).

[5] ESTRADA, M. Fernanda, SÁ, Carlos C., QUEIRÓ, João F., SILVA, M. Céu, COSTA, M. José, História da Matemática. Lisboa, Universidade Aberta, 2000.

[6] FABER, Richard L., Foundations of Euclidean and Non-Euclidean Geometry. - New York, Marcel Dekker, Inc., 1983.

[7] KLINE, Morris, Mathematical Thought: From Ancient to Modern Times, Volume 1. Oxford, Oxford University Press, 1990 (ed. orig.: 1972).

[8] KLINE, Morris, Mathematical Thought: From Ancient to Modern Times, Volume 3. Oxford, Oxford University Press, 1990 (ed. orig.: 1972).

[9] MLODINOW, Leonard, Euclid's Window. London, Penguin Books, 2002 (ed. orig.: Free Press, 2001).

[10] SOUSA, Cristina A. S., O Erro de Euclides (A Presença das Geometrias Não Euclidianas na Natureza e na Arte e a Sua Ausência nos Planos de Estudos do Ensino Não Superior e Superior), Universidade Portucalense Infante D. Henrique, Porto, 2009. 